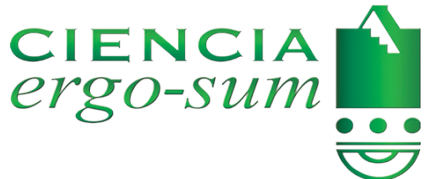

CIENCIA ergo-sum

Universidad Autónoma del Estado de México

ciencia.ergosum@yahoo.com.mx

E-ISSN : 2395-8782

\title{
Tortilla de maíz adicionada con harina de Brosimum alicastrum: propiedades fisicoquímicas y actividad antioxidante
}

Moo-Huchin, Víctor Manuel; Góngora-Chi, Guadalupe J ohanna; Sauri-Duch, Enrique; Canto-Pinto, J orge Carlos; Betancur-Ancona, David; Ramón-Canul, Lorena Guadalupe

Tortilla de maíz adicionada con harina de Brosimum alicastrum: propiedades fisicoquímicas y actividad antioxidante

CIENCIA ergo-sum, vol. 28, núm. 3, noviembre 2021-febrero 2022 | e129

Universidad Autónoma del Estado de México, México

Esta obra está bajo una Licencia Creative Commons Atribución-NoComercial-SinDerivar 4.0 Internacional.

Moo-Huchin, V. M., Góngora-Chi, G. J ., Sauri-Duch, E., Canto-Pinto, J . C., Betancur-Ancona, D. y Ramón-Canul, L. G. (2021). Tortilla de maíz adicionada con harina de Brosimum alicastrum: propiedades fisicoquímicas y actividad antioxidante. CIENCI A ergo-sum, 28(3). https://doi.org/10.30878/ces.v28n3a1 


\title{
Tortilla de maíz adicionada con harina de Brosimum alicastrum: propiedades fisicoquímicas y actividad antioxidante
}

\section{Corn Tortilla added with Brosimum alicastrum flour: Physicochemical properties and antioxidant activity}

Victor Manuel Moo-Huchin

Tecnológico Nacional de México-Campus Mérida, México

vmmoo@yahoo.com

(D) https://orcid.org/0000-0002-9365-8922

Recepción: 28 de enero de 2020

Guadalupe Johanna Góngora-Chi

Tecnológico Nacional de México-Campus Mérida, México

ln.johanna@hotmail.com

(D) https://orcid.org/0000-0002-9738-2679

Enrique Sauri-Duch

Tecnológico Nacional de México-Campus Mérida, México

esauri5@yahoo.com.mx

(D) https://orcid.org/0000-0003-2181-8592

Jorge Carlos Canto-Pinto

Tecnológico Nacional de México-Campus Calkini, México

jccanto@itescam.edu.mx

(D) https://orcid.org/0000-0002-6133-056X

David Betancur-Ancona

Universidad Autónoma de Yucatán, México

bancona@correo.uady.mx

(D) https://orcid.org/0000-0002-9206-3222

Lorena Guadalupe Ramón-Canul

Universidad de la Sierra Sur Oaxaca, México

1_g_r_c@hotmail.com

(1) https://orcid.org/0000-0002-1974-3256

\section{ReSUMEN}

Se obtienen tortillas a partir de harina de maíz nixtamalizado, al cual se le adicionaron diferentes proporciones de harina de $B$. alicas$\operatorname{trum}(0,10,20$ y $30 \% \mathrm{p} / \mathrm{p}$ en base seca). Se analizan las tortillas en relación con la composición química, perfil de aminoácidos, compuestos fenólicos, actividad antioxidante y propiedades físicas. La adición de harina de $B$. alicastrum en tortillas aumentó la actividad antioxidante, el contenido de minerales, fibra soluble, proteína, triptófano, compuestos fenólicos totales y flavonoides totales comparado con la tortilla control. Los resultados sugieren que la adición de harina de B. alicastrum ( 10 y $20 \%$ ) a la harina de maíz mejoró el valor nutricional y funcional de tortillas sin afectar su calidad (rolabilidad, grado de inflado y pérdida por cocción).

Palabras Claves: B. alicastrum, antioxidantes, masa, tortillas.

\begin{abstract}
Tortillas were obtained using nixtamalized corn flour with $B$. alicastrum flour at different proportions $(0,10,20 \mathrm{y} 30 \%$, w/w on a dry basis). Tortillas were analyzed in relation to the chemical composition, amino acid profile, phenolic compounds, antioxidant activity and physical properties. The addition of $B$. alicastrum flour in tortillas increased the antioxidant activity, ash content, soluble dietary fiber, total proteins, tryptophan, phenolic compounds and total flavonoids compared to the control tortilla. The results suggest that the addition of $B$. alicastrum flour (10 and $2 \%$ ) to corn flour increased the nutritional and functional value in tortillas while maintaining their quality (rollability, puffiness and cooking loss).
\end{abstract}

KEYWORDS: B. alicastrum, antioxidants, dough, tortillas. 


\section{INTRODUCCIÓN}

En México se reconoce a la tortilla como un alimento plano, delgado y redondo, el cual se elabora a partir de harina de maíz nixtamalizada (que consiste en la cocción de granos de maíz en hidróxido de calcio) o de trigo (Liu et al., 2016); sin embargo, el proceso para producirlas y las características de calidad de ambas son distintas. Las tortillas de maíz se obtienen a partir de masas pregelatinizadas y cohesivas, mientras que las tortillas de trigo de masas con gluten con alto contenido de grasa (Saldívar y Chuck-Hernández, 2016). La tortilla de maíz es un alimento básico consumido comúnmente por la población urbana y rural en México (consumo per cápita de $75 \mathrm{~kg}$ por año) que la prefiere fresca debido a su textura suave, pues permite enrollarla, doblarla sin romperse y rellenarla en su interior con alimentos como carne, frijoles, queso, etc. Es considerada como una buena fuente de energía en la dieta mexicana, característica atribuida por su alto contenido de almidón, pero deficiente en dos aminoácidos esenciales (lisina y triptófano); por esta razón, se han desarrollado estrategias para mejorar el contenido nutrimental de la tortilla como el uso de variedades de maíz con alto contenido de estos aminoácidos (Bello-Pérez et al., 2016) o la adición de harinas no tradicionales (ricos en nutrientes y compuestos bioactivos) durante su elaboración. Las tortillas se consideran un vehículo ideal para la fortificación de nutrientes y adición de compuestos con actividad antioxidante; sin embargo, se ha observado que al hacerlo resultan afectadas las características de calidad de las tortillas (suavidad, rolabilidad y grado de inflado) (Chuck-Hernández et al., 2015; Treviño-Mejía et al., 2016). Para mejorar sus propiedades nutricionales, las tortillas de maíz se han fortificado por medio de la adición de frijol (Phaseolus vulgaris), harina de hojas de Moringa oleifera, Salvia hispánica y amaranto (Amaranthus spp.) (Rendón-Villalobos et al., 2012, Anton et al., 2008, Páramo-Calderón et al., 2019; Vázquez-Rodríguez et al., 2013). El uso de harinas no tradicionales para mejorar las propiedades nutricionales y funcionales de la tortilla de maíz sin afectar su calidad como la rolabilidad y el grado de inflado es relevante en la industria de la masa y tortilla.

B. alicastrum es un árbol tropical cuyo registro histórico indica que tuvo un gran impacto en la época precolombina, cuando los antiguos pueblos nativos mayas de la península de Yucatán utilizaban sus semillas como un alimento alternativo en caso de que sequías o plagas afectaran los cultivos habituales como el maíz (Moo-Huchin et al., 2019). Las frutas del árbol de B. alicastrum se consumían crudos e incluso sus semillas secas y molidas se mezclaban con maíz para hacer tortillas. Se sabe que las semillas tienen un alto valor nutricional, caracterizado por su mayor contenido de triptófano en comparación con la avena, frijoles pintos, harina de maíz y arroz (GRAS report, 2007). Pérez-Pacheco et al. (2014), Ríos-Soberanis et al. (2016) y Moo-Huchin et al. (2015) han reportado las propiedades del almidón de las semillas de $B$. alicastrum para su uso en la industria alimentaria, pero existen pocos estudios sobre el contenido de compuestos bioactivos de las semillas y su actividad biológica. La harina de semillas de $B$. alicastrum tiene mayor contenido de compuestos fenólicos y actividad antioxidante cuando se compara con la harina de nueces tales como maní y almendra (Ozer, 2017). Recientemente, la harina de $B$. alicastrum ha despertado el interés por su contenido de ácidos fenólicos y flavonoides tales como ácido gálico, ácido clorogénico, ácido cafeico, epicatequina, catequina, ácido vanílico, ácido sinápico, ácido ferúlico, quercetina y ácido trans-cinámico (Ozer, 2017, Moo-Huchin et al., 2019). Esta información ha contribuido a caracterizar a la harina y a valorar su uso como ingrediente o tal cual como harina funcional para producir tortillas de trigo (Subiria-Cueto et al., 2019). Domínguez-Zarate et al. (2019) reportaron algunas particularidades (textura y color) de tortillas de maíz elaboradas con harina de $B$. alicastrum para aumentar el contenido de fibra dietética; sin embargo, se requiere una caracterización más detallada.

De acuerdo con este contexto, el objetivo de este artículo es caracterizar las propiedades físicas, nutricionales y antioxidantes de tortillas de maíz enriquecidas con harina de semillas de B. alicastrum. 


\section{Materiales y métodos}

\section{1. Obtención de harina}

Se utilizó harina comercial de maíz nixtamalizado (Maseca $\left.{ }^{\circledR}\right)$, la cual se compró en un supermercado de Mérida, Yucatán, México. Por otra parte, los frutos del árbol de B. alicastrum se recolectaron en este mismo estado, durante 2019. Los frutos se seleccionaron de acuerdo con el color (amarillo-naranja) y se pelaron manualmente (removiendo la testa) para sacar las semillas. El secado de las semillas, molienda y reducción de tamaño de partícula para conseguir la harina fina se realizó de acuerdo con lo reportado por Pérez-Pacheco et al. (2014). La harina resultante se almacenó en bolsas de polietileno a $4.0 \pm 1.0^{\circ} \mathrm{C}$ hasta su uso.

\subsection{Obtención de tortillas}

Se obtuvieron tres mezclas de harinas a diferentes proporciones de harina de $B$. alicastrum y harina de maíz nixtamalizada (0:100, 10:90, 20:80, 30:70,p/p) utilizando una mezcladora Kitchen Aid ${ }^{\circledR}$ (Kitchen Aid Inc. St. Joseph, USA) con mezclador tipo paleta. La harina comercial de maíz nixtamalizada se consideró como tratamiento control. Las mezclas y la harina control fueron evaluadas en relación con el índice de solubilidad y el índice de absorción de agua. En estudios preliminares se encontró que una incorporación superior a $30 \%$ de harina de $B$. alicastrum no permite la formación de discos planos de masa en la máquina de tortillas.

Para elaborar las tortillas, cada mezcla y la harina control se hidrataron con agua potable a $25^{\circ} \mathrm{C}$ a un contenido de humedad de $58 \%$ (calculado por medio de un balance de materia) y se amasó manualmente durante 5 min hasta crear una masa de buena consistencia: aquella que al presionarla entre dos placas metálicas cubiertas de plástico, hasta un espesor de $2-3 \mathrm{~mm}$, pueda despegarse sin romperse (Gasca-Mancera y Casas-Alencáster, 2007).

La masa se colocó en los rodillos de la máquina de tortillas (Tecnomaiz S. A. de C. V., Grupo Gruma), la cual se calibró previamente a $1.11 \mathrm{~mm}$ para lograr que todas fueran del mismo espesor. Los discos de masa se sometieron a cocción al pasar en el horno de la máquina, precalentado a $280{ }^{\circ} \mathrm{C}$, y después de 2 min salieron las tortillas (figura 1). Las tortillas se enfriaron, liofilizaron, molieron y por último el polvo resultante se almacenó a $-20^{\circ} \mathrm{C}$ en bolsas de polietileno para su análisis.

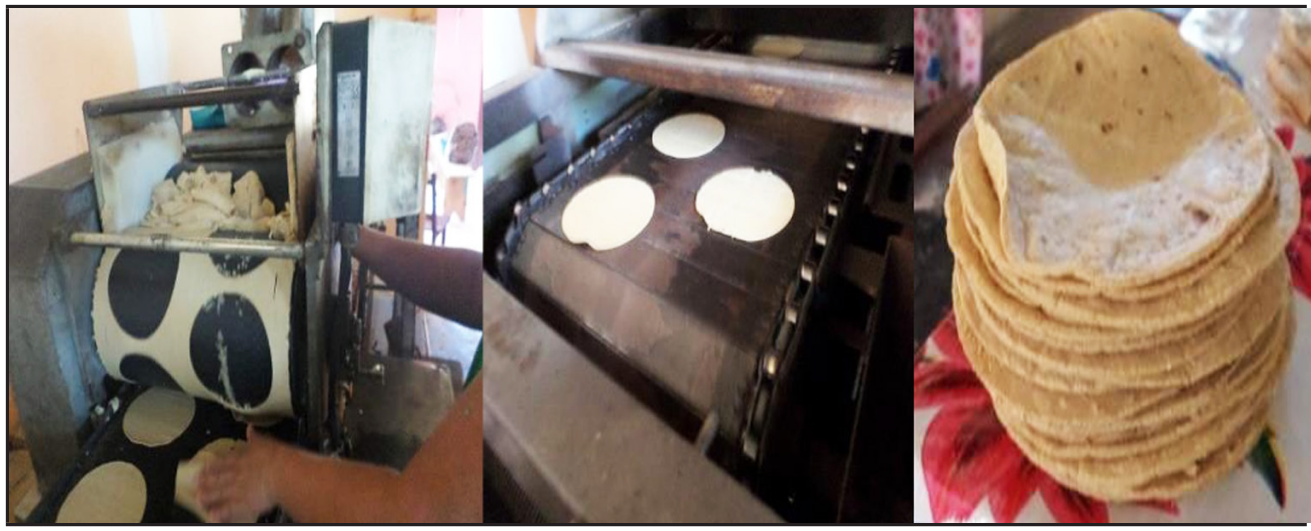

FIGURA 1

Proceso de elaboración de tortillas de maíz adicionado con $10 \%$ harina de $B$. alicastrum Fuente: fotografías propias. 


\subsection{Análisis de las mezclas de harina}

\section{3. 1. Indice de solubilidad en agua (ISA) y absorción de agua (IAA)}

Los índices ISA e IAA se obtuvieron de acuerdo con el método descrito por Anderson (1982) utilizando la fórmula (g de precipitado/g muestra seca) para IAA y (g sólidos solubles/g muestra seca) *100 para ISA.

\subsection{Análisis de tortillas}

\section{4. 1. Composición química}

Se determinó la composición química del polvo de tortillas siguiendo la metodología descrita por la AOAC para humedad (925.10), cenizas (923.03), proteínas (920.87) y lípidos (920.39) (AOAC, 1997). Para determinar el contenido de fibra dietética (total, soluble e insoluble), se empleó un procedimiento enzimático-gravimétrico reportado por Pérez-Pacheco et al. (2014).

\section{4. 2. Análisis de aminoácidos}

El análisis de aminoácidos de las tortillas se realizó con un sistema HPLC-1220 Infinity (Agilent Technologies, Palo Alto, CA., USA) con detección espectrofotométrica a $280 \mathrm{~nm}$. Se utilizó como estándar interno el ácido L-aminobutírico. La información de las características de la columna, hidrólisis de la muestra, derivatización, composición y flujo de la fase móvil utilizado en este trabajo ha sido descrito por Ruiz-Ruiz et al. (2008). Los resultados se expresaron como g del aminoácido/100 g proteína.

\section{4. 3. Propiedades físicas}

Se determinó la pérdida de peso en la cocción (\%) calculando el porcentaje en peso de material que se pierde durante el cocimiento; se pesaron seis tortillas antes y después de someterlas a la cocción $\left(280^{\circ} \mathrm{C}\right)$.

El diámetro $(\mathrm{cm})$ y el espesor $(\mathrm{mm})$ de seis tortillas se midieron con una regla y un medidor de espesores de precisión respectivamente.

La rolabilidad y el grado de inflado (formación de ampolla) de seis tortillas se llevó a cabo de acuerdo con lo reportado por Treviño-Mejía et al. (2016).

Se realizó el ensayo de tensión de nueve tortillas por tratamiento. Previo a la medición, las tortillas se cortaron (de la parte central) en forma de rectángulos de $2 \mathrm{~cm}$ de ancho por $10 \mathrm{~cm}$ de largo para obtener las probetas. Las probetas se sujetaron con dos pinzas de tensión del equipo Instron Universal Testing Machine (Model 4411, Canton, MA, USA). El ensayo se realizó a una velocidad de $120 \mathrm{~mm} / \mathrm{min}$ y una distancia de $60 \mathrm{~mm}$. A partir de las curvas alcanzadas, se calculó la resistencia a la ruptura $(\mathrm{KPa})$, porcentaje de elongación (\%) y el módulo de Young’s $(\mathrm{KPa})$.

Los parámetros $\mathrm{L}^{*}$ (luminosidad), $\mathrm{a}^{*}\left(+\mathrm{a}^{*}=\right.$ rojo, $-\mathrm{a}^{*}=$ verde $)$ y $\mathrm{b}^{*}\left(+\mathrm{b}^{*}=\right.$ amarillo, $\left.-\mathrm{b}^{*}=\mathrm{azul}\right)$ se determinó con un colorímetro (Hunter Lab MiniScan modelo No. XE Plu, Reston, Virginia). La medición se hizo en tres regiones distintas sobre la superficie de cada tortilla (seis tortillas por tratamiento). Previo a este proceso, se utilizó una placa blanca de calibración del equipo. Con los valores obtenidos se calculó el ángulo de tono $\left({ }^{\circ} \mathrm{Hue}\right)$ y cromaticidad $\left(\mathrm{C}^{*}\right)$.

\section{4. 4. Compuestos fenólicos totales (CFT), flavonoides totales (FT) y actividad antioxidante}

Para la extracción de antioxidantes, $1 \mathrm{~g}$ de polvo de tortillas se mezcló con $9 \mathrm{~mL}$ de metanol absoluto a $100 \mathrm{rpm}$ durante $2 \mathrm{~h}$. El extracto fue centrifugado a $2500 \mathrm{rpm}$ por $5 \mathrm{~min}$; una vez recolectado el sobrenadante y el 
sedimento resultante (pellet), se sometió a una nueva extracción con el mismo procedimiento. Los sobrenadantes de ambas extracciones se juntaron y finalmente se almacenaron a $-20{ }^{\circ} \mathrm{C}$ bajo condiciones de obscuridad.

La determinación del contenido de CFT (reactivo de Folin-Ciocalteu's) y FT (cloruro de aluminio) de los extractos de las tortillas se efectuó de acuerdo con lo descrito por Moo-Huchin et al. (2014). Ambos métodos se basan en mediciones espectrofotométricas $(765 \mathrm{~nm}$ para CFT y $415 \mathrm{~nm}$ para FT) utilizando un espectrofotómetro UV-Vis Angilet Technologies Cary 60. Los resultados se expresaron como mg equivalentes de ácido gálico (EAG)/100 g de polvo seco (PS) para CFT y mg equivalentes de quercetina (EQ)/100 g PS para FT.

La actividad antioxidante de los extractos de las tortillas se determinó utilizando el ensayo DPPH ( $\mu \mathrm{M}$ trolox/100 g PS) (Moo-Huchin et al., 2014) y poder reductor (mg de ácido ascórbico/100 g PS) (Can-Cauich et al., 2017).

\section{5. Análisis estadístico}

Los resultados se sometieron a un análisis de varianza de una vía. Se utilizó la prueba de rangos múltiples Tukey’s a un nivel de significancia de $P \leq 0.05$ para identificar diferencias significativas en los promedios de los parámetros medidos.

\section{Resultados Y Discusión}

\section{1. Índices de absorción de agua (IAA) y solubilidad en agua (ISA) de harinas}

En el cuadro 1 se reportan los valores de los índices IAA e ISA de harina de maíz nixtamalizado $\left(\mathrm{M}_{0}\right)$ y harina de maíz nixtamalizado adicionado con diferentes proporciones de $B$. alicastrum $\left(\mathrm{M}_{1}=10 \%, \mathrm{M}_{2}=20 \%\right.$ y $\mathrm{M}_{3}=$ $30 \%)$. Cuando se incorporó el $20\left(\mathrm{M}_{2}\right)$ y $30 \%\left(\mathrm{M}_{3}\right)$ de harina de $B$. alicastrum a $\mathrm{M}_{0}$ se logró una disminución de los valores de IAA; este hecho se puede atribuir a la presencia de hidrocoloides (ejemplo, las gomas) en la harina comercial que es adicionada para mejorar las propiedades de textura de la masa y tortillas y además favorece la retención de humedad para que las tortillas se mantengan frescas (Flores-Farías et al., 2002). Asimismo, las diferencias en el valor de IAA estarían relacionadas con el tamaño de partícula de las harinas o el grado de gelatinización del almidón en donde a una mayor gelatinización da lugar a harinas con altas tasas de absorción de agua (Contreras-Jiménez et al., 2014).

El valor de IAA está muy asociado a la suavidad, dureza o sequedad de la tortilla, así como al número y al peso de tortillas por $100 \mathrm{~g}$ de harina, es decir, qué harinas con valores altos de IAA son las que dan mayor número y peso de tortillas. Acorde con los resultados, se recomienda incorporar hidrocoloides a las mezclas que contengan harina de $B$. alicastrum para favorecer la retención de la humedad.

Por otra parte, el valor de ISA indica la cantidad de sólidos disueltos en el agua de una harina que es expuesta a un exceso de este líquido y también indica el grado de cocción del producto (Flores-Farías et al., 2002). En este trabajo, los valores de ISA para $\mathrm{M}_{0}, \mathrm{M}_{1}, \mathrm{M}_{2}$ y $\mathrm{M}_{3}$ resultaron con una variabilidad entre $4.38 \pm 0.16$ y $10.41 \pm$ $0.06 \%$. A medida que se incrementó la adición de harina de $B$. alicastrum a $\mathrm{M}_{0}$, también aumentó el valor de ISA. Este resultado probablemente sea atribuido a la lixiviación de las sales minerales en el medio acuoso o por la disolución de polisacáridos solubles en agua contenidos en la harina de B. alicastrum.

\section{2. Composición química de las tortillas}

Este artículo reporta la composición química de tortillas hechas con harina de maíz nixtamalizado (control) y harina de maíz nixtamalizado adicionado con 10, 20 y 30\% de harina de B. alicastrum (T-10, T-20 y T-30 respectivamente) (cuadro 2). Con base en los resultados, la composición química resultó diferente entre las tortillas. 
La tortilla control resultó con mayor contenido de humedad (41.9 $\pm 0.9 \%)$ cuando se compara con T-10, T-20 y T-30. Este hecho es explicado por la variación de los valores IAA de las harinas utilizadas para hacer tortillas (cuadro 1).

\section{CUADRO 1}

Índices IAA e ISA de harina comercial de maíz nixtamalizado y mezclas con harina de $B$. alicastrum

\begin{tabular}{|ccc|}
\hline Muestra & $\begin{array}{c}\text { IAA } \\
\text { (g agua/g harina seca) }\end{array}$ & ISA (\%) \\
\hline $\mathrm{M}_{0}$ & $3.41 \pm 0.00 \mathrm{~b}$ & $4.38 \pm 0.16 \mathrm{a}$ \\
$\mathrm{M}_{1}$ & $3.26 \pm 0.07 \mathrm{ab}$ & $7.30 \pm 0.21 \mathrm{~b}$ \\
$\mathrm{M}_{2}$ & $3.21 \pm 0.00 \mathrm{a}$ & $8.72 \pm 0.00 \mathrm{c}$ \\
$\mathrm{M}_{3}$ & $3.19 \pm 0.03 \mathrm{a}$ & $10.41 \pm 0.06 \mathrm{~d}$ \\
\hline
\end{tabular}

Fuente: elaboración propia.

Nota: $\mathrm{M}_{0}=$ harina de maíz nixtamalizado; $\mathrm{M}_{1}=$ mezcla harina de maíz nixtamalizado + harina de $B$ alicastrum $(90: 10) ; \mathrm{M}_{2}=\mathrm{Mezcla}$ harina de maíz nixtamalizado + harina de B. alicastrum (80:20) y $\mathrm{M}_{3}=$ mezcla harina de maíz nixtamalizado + harina de $B$. alicastrum (70:30). Los resultados son promedios de tres repeticiones \pm desviación estándar; valores con diferentes letras dentro de cada columna son significativamente diferentes a $P \leq 0.05$.

La adición de $30 \%$ de harina de $B$. alicastrum produjo tortillas con mayor contenido de cenizas $(1.23 \pm$ $0.06 \%)$ y fibra soluble $(1.93 \pm 0.04 \%)$ en comparación al control, T-10 y T-20. Las tortillas T-20 y T-30 consiguieron mayor contenido de proteína total respecto al control y T-10. Esta diferencia es atribuida a la composición química de la harina de $B$. alicastrum, considerada fuente de proteínas y elementos minerales (Pérez-Pacheco et al., 2014). La composición química de las tortillas T-10, T-20, T-30 y control es comparable a lo reportado para tortillas de maíz suplementado con semilla de chía y frijol (Rendón-Villalobos et al., 2012; Treviño-Mejía et al., 2016).

En este sentido, Subiria-Cueto et al. (2019) reportaron que la incorporación de harina de B. alicastrum (25\%) incrementó el contenido de fibra dietética, cobre y potasio de tortillas de trigo. En otro trabajo, se sugiere utilizar $10 \%$ de harina de $B$. alicastrum Maya óox (adquirido comercialmente) en tortillas de maíz para que aumente el contenido de fibra dietética (Domínguez-Zárate et al., 2019).

\section{CUADRO 2}

Composición química (expresada en base húmeda) de tortillas de maíz adicionado con harina de B. alicastrum en diferentes niveles

\begin{tabular}{|lccccccc}
\hline Muestra & $\begin{array}{c}\text { Humedad } \\
(\%)\end{array}$ & $\begin{array}{c}\text { Cenizas } \\
(\%)\end{array}$ & $\begin{array}{c}\text { Lípidos } \\
(\%)\end{array}$ & $\begin{array}{c}\text { Fibra soluble } \\
(\%)\end{array}$ & $\begin{array}{c}\text { Fibra insoluble } \\
(\%)\end{array}$ & $\begin{array}{c}\text { Fibra dietética } \\
\text { total }(\%)\end{array}$ & $\begin{array}{c}\text { Proteínas } \\
(\%)\end{array}$ \\
\hline Control & $41.9 \pm 0.9 \mathrm{~b}$ & $0.75 \pm 0.04^{\mathrm{a}}$ & $1.10 \pm 0.05 \mathrm{a}$ & $0.97 \pm 0.00 \mathrm{a}$ & $8.46 \pm 0.04 \mathrm{~b}$ & $9.43 \pm 0.04 \mathrm{a}$ & $4.62 \pm 0.00 \mathrm{a}$ \\
$\mathrm{T}-10$ & $38.4 \pm 01 \mathrm{a}$ & $0.97 \pm 0.04 \mathrm{~b}$ & $1.13 \pm 0.04 \mathrm{a}$ & $1.26 \pm 0.00 \mathrm{~b}$ & $9.07 \pm 0.04 \mathrm{c}$ & $10.33 \pm 0.04 \mathrm{c}$ & $5.14 \pm 0.04 \mathrm{~b}$ \\
$\mathrm{~T}-20$ & $38.15 \pm 00 \mathrm{a}$ & $1.11 \pm 0.00 \mathrm{c}$ & $1.15 \pm 0.05 \mathrm{a}$ & $1.71 \pm 0.04 \mathrm{c}$ & $8.27 \pm 0.04 \mathrm{a}$ & $9.95 \pm 0.04 \mathrm{~b}$ & $5.34 \pm 0.04 \mathrm{c}$ \\
$\mathrm{T}-30$ & $39.2 \pm 11 \mathrm{a}$ & $1.23 \pm 0.06 \mathrm{~d}$ & $1.11 \pm 0.06 \mathrm{a}$ & $1.93 \pm 0.04 \mathrm{~d}$ & $8.30 \pm 0.04 \mathrm{ab}$ & $10.20 \pm 0.04 \mathrm{c}$ & $5.42 \pm 0.04 \mathrm{c}$ \\
\hline
\end{tabular}

Fuente: elaboración propia.

Nota: $\mathrm{T}-10=$ tortilla $+10 \%$ harina $\mathrm{B}$. alicastrum; $\mathrm{T}-20=$ tortilla $+20 \%$ harina $B$. alicastrum; $\mathrm{T}-30=$ tortilla $+30 \%$ harina B. alicastrum. Los resultados son promedios de tres repeticiones \pm desviación estándar; valores con diferentes letras dentro de cada columna son significativamente diferentes a $P \leq 0.05$.

El perfil de aminoácidos es uno de los parámetros más importantes dentro de la evaluación de las tortillas adicionadas con harina de $B$. alicastrum. Los productos a base de cereales (como el caso de las tortillas) tienen lisina como el aminoácido más limitante, seguido del triptófano (Chuck-Hernández et al., 2015). La incorporación 
de harina de $B$. alicastrum afectó el contenido de aminoácidos (g/100 g de proteína) de las tortillas de maíz (cuadro 3). En la mayoría de los casos, las tortillas adicionadas con harina de B. alicastrum lograron un mayor contenido de aminoácidos respecto al control. Acorde con los requerimientos mínimos de aminoácidos esenciales según la FAO/WHO (1991) para infantes y preescolares se obtuvieron valores superiores para histidina (T-10), valina (T-20 y T-30), isoleucina (T-20 y T-30) y leucina (T-20 y T-30). El triptófano es considerado un aminoácido limitante en las tortillas de maíz nixtamalizado tradicionalmente y cubre $70 \%$ del requerimiento según la FAO/WHO (Waliszewski et al., 2002). Tras la adición de harina de $B$. alicastrum a las tortillas, el contenido de triptófano aumentó respecto al control, por lo que excedió los requerimientos de este aminoácido para infantes según la FAO/OMS. La lisina y treonina fueron los aminoácidos limitantes para todas las tortillas al considerar un requerimiento para infantes de 5.8 y $3.4 \mathrm{~g} / 100 \mathrm{~g}$ proteína respectivamente. La variación de los valores del contenido de aminoácidos en tortillas adicionadas con harina de B. alicastrum sería causado por las interacciones entre los aminoácidos y los componentes de la matriz de las mezclas de harinas y presentar cambios en las estructuras de las proteínas y a su vez formar complejos entre proteína-almidón, proteína-hemicelulosa y proteína-minerales (Ruíz-Ruíz et al., 2008).

\section{CUADRO 3}

Composición de aminoácidos de tortillas de maíz adicionado con harina de B. alicastrum en diferentes niveles y requerimientos de la FAO (g/100 g proteína)

\begin{tabular}{lrrrrr}
\hline Aminoácido & \multicolumn{1}{c}{ Control } & \multicolumn{1}{c}{ T-10 } & \multicolumn{1}{c}{ T-20 } & T-30 & $\begin{array}{c}\text { g/100 g proteína } \\
\text { (FAO/WHO) }\end{array}$ \\
\hline Ácido aspártico & $0.82 \pm 0.04^{\mathrm{a}}$ & $3.13 \pm 0.21 \mathrm{~d}$ & $2.46 \pm 0.02 \mathrm{c}$ & $2.34 \pm 0.02 \mathrm{~b}$ & \\
Ácido glutámico & $1.84 \pm 0.05^{\mathrm{a}}$ & $3.69 \pm 0.14 \mathrm{c}$ & $2.64 \pm 0.07 \mathrm{~b}$ & $4.12 \pm 0.19 \mathrm{~d}$ & \\
Serina & $1.77 \pm 0.15 \mathrm{~b}$ & $3.37 \pm 0.08 \mathrm{~d}$ & $1.65 \pm 0.04 \mathrm{a}$ & $1.90 \pm 0.03 \mathrm{c}$ & \\
Histidina & $1.42 \pm 0.12 \mathrm{c}$ & $2.16 \pm 0.04 \mathrm{~d}$ & $0.96 \pm 0.02 \mathrm{a}$ & $1.17 \pm 0.08 \mathrm{~b}$ & 1.9 \\
Glicina & $1.14 \pm 0.03 \mathrm{~b}$ & $1.78 \pm 0.08 \mathrm{~d}$ & $1.10 \pm 0.09 \mathrm{a}$ & $1.21 \pm 0.03 \mathrm{c}$ & \\
Treonina & $1.61 \pm 0.01 \mathrm{~b}$ & $2.74 \pm 0.53 \mathrm{~d}$ & $1.40 \pm 0.05 \mathrm{a}$ & $1.64 \pm 0.03 \mathrm{c}$ & 3.4 \\
Arginina & $16.30 \pm 0.80 \mathrm{a}$ & $26.43 \pm 1.43 \mathrm{~d}$ & $16.63 \pm 0.64 \mathrm{~b}$ & $16.89 \pm 0.10 \mathrm{c}$ & \\
Alanina & $1.62 \pm 0.01 \mathrm{~b}$ & $2.54 \pm 0.36 \mathrm{~d}$ & $1.46 \pm 0.07 \mathrm{a}$ & $1.85 \pm 0.00 \mathrm{c}$ & \\
Prolina & $23.80 \pm 0.82 \mathrm{c}$ & $25.61 \pm 1.58 \mathrm{~d}$ & $12.43 \pm 0.72 \mathrm{a}$ & $13.53 \pm 0.28 \mathrm{~b}$ & \\
Tirosina & $2.17 \pm 0.02 \mathrm{c}$ & $3.21 \pm 0.83 \mathrm{~d}$ & $1.49 \pm 0.07 \mathrm{a}$ & $2.02 \pm 0.12 \mathrm{~b}$ & \\
Valina & $9.22 \pm 0.83 \mathrm{~b}$ & $5.20 \pm 0.63 \mathrm{a}$ & $13.05 \pm 0.18 \mathrm{~d}$ & $11.20 \pm 0.04 \mathrm{c}$ & 3.5 \\
Metionina & $0.73 \pm 0.09 \mathrm{~b}$ & $0.25 \pm 0.06 \mathrm{a}$ & $1.00 \pm 0.18 \mathrm{~d}$ & $0.89 \pm 0.00 \mathrm{c}$ & 2.5 \\
Cisteína & $20.92 \pm 0.87 \mathrm{~b}$ & $11.85 \pm 1.29 \mathrm{a}$ & $29.63 \pm 2.27 \mathrm{~d}$ & $25.41 \pm 0.08 \mathrm{c}$ & \\
Isoleucina & $3.22 \pm 0.09 \mathrm{c}$ & $1.44 \pm 0.06 \mathrm{a}$ & $3.13 \pm 0.01 \mathrm{~b}$ & $3.58 \pm 0.08 \mathrm{~d}$ & 2.8 \\
Leucina & $9.55 \pm 0.41 \mathrm{~d}$ & $4.03 \pm 0.03 \mathrm{a}$ & $7.62 \pm 0.36 \mathrm{~b}$ & $8.38 \pm 0.75 \mathrm{c}$ & 6.6 \\
Fenilalanina & $2.78 \pm 0.06 \mathrm{~d}$ & $0.98 \pm 0.07 \mathrm{a}$ & $2.14 \pm 0.06 \mathrm{~b}$ & $2.65 \pm 0.07 \mathrm{c}$ & 3.6 \\
Lisina & $1.01 \pm 0.03 \mathrm{a}$ & $1.51 \pm 0.17 \mathrm{~d}$ & $1.12 \pm 0.00 \mathrm{~b}$ & $1.14 \pm 0.01 \mathrm{c}$ & 5.8 \\
Triptófano & $0.72 \pm 0.01 \mathrm{a}$ & $1.45 \pm 0.03 \mathrm{~b}$ & $1.62 \pm 0.03 \mathrm{c}$ & $1.92 \pm 0.03 \mathrm{~d}$ & 1.1 \\
\hline & & $F$ & &
\end{tabular}

Fuente: elaboración propia.

Nota: $\mathrm{T}-10=$ tortilla $+10 \%$ harina $B$. alicastrum; $\mathrm{T}-20=$ tortilla $+20 \%$ harina $B$. alicastrum; $\mathrm{T}-30=$ tortilla $+30 \%$ harina $\mathrm{B}$. alicastrum . Los resultados son promedios de tres repeticiones \pm desviación estándar; valores con diferentes letras en cada fila son significativamente diferentes a $P \leq 0.05$. 


\section{3. Contenido de CFT, FT y actividad antioxidante}

La actividad antioxidante de una matriz alimentaria es determinada por las interacciones entre los diferentes compuestos bioactivos como los ácidos fenólicos y flavonoides, lo cual tienen diferentes mecanismos de reacción para retrasar o inhibir el deterioro oxidativo causado por radicales libres (Moo-Huchin et al., 2019). En lo referente a este artículo, la adición de harina de $B$. alicastrum mejoró significativamente el contenido de CFT, FT y la actividad antioxidante de las tortillas de maíz (cuadro 4). Las tortillas adicionadas con harina de $B$. alicastrum aumentaron entre 4.0 y 8.0 veces el contenido de CFT, 1.3 veces el contenido de FT, entre 1.1 y 1.3 veces la actividad antioxidante DPPH y entre 2.0 y 3.3 veces la actividad antioxidante poder reductor $\left(\mathrm{Fe}^{3+}\right.$ se reduce a $\mathrm{Fe}^{2+}$ ). En este sentido, Moo-Huchin et al. (2019) indican que la harina de B. alicastrum tiene alto contenido de ácido gálico, ácido clorogénico y ácido vanílico que contribuyen en su actividad antioxidante. Este hecho explica el mejoramiento de las propiedades antioxidantes de las tortillas de maíz. Por lo tanto, la harina de B. alicastrum sería una alternativa para que las tortillas otorguen un valor agregado y, además, se considere como un alimento funcional que ofrezca beneficios para la salud.

\section{CUADRO 4}

Contenido de CFT, FT y actividad antioxidante de tortillas de maíz adicionado con harina de B. alicastrum en diferentes niveles

\begin{tabular}{|c|c|c|c|c|}
\hline \multirow[t]{2}{*}{ Muestra } & \multirow{2}{*}{$\begin{array}{c}\text { Compuestos fenólicos } \\
\text { totales }\end{array}$} & \multirow{2}{*}{$\begin{array}{l}\text { Flavonoides totales } \\
\text { (mg EQ/100 g PS) }\end{array}$} & \multicolumn{2}{|c|}{ Actividad antioxidante } \\
\hline & & & $\begin{array}{c}\mathrm{DPPH}(\mu \mathrm{M} \\
\text { trolox } / 100 \mathrm{~g} \mathrm{PS})\end{array}$ & $\begin{array}{l}\text { Poder reductor (mg ácido } \\
\text { ascórbico/100 g PS) }\end{array}$ \\
\hline Control & $85.09 \pm 5.77 \mathrm{a}$ & $71.47 \pm 0.64 a$ & $314.72 \pm 20.68 \mathrm{a}$ & $63.18 \pm 3.77 \mathrm{a}$ \\
\hline $\mathrm{T}-10$ & $368.42 \pm 1.92 b$ & $95.88 \pm 1.28 b$ & $351.48 \pm 15.24 b$ & $138.95 \pm 2.74 b$ \\
\hline $\mathrm{T}-20$ & $641.23 \pm 57.66 c$ & $98.24 \pm 1.28 \mathrm{c}$ & $408.04 \pm 8.71 \mathrm{c}$ & $179.70 \pm 4.11 \mathrm{c}$ \\
\hline $\mathrm{T}-30$ & $684.65 \pm 20.18 d$ & $98.53 \pm 0.64 c$ & $409.92 \pm 10.88 c$ & $208.98 \pm 14.05 \mathrm{~d}$ \\
\hline
\end{tabular}

Fuente: elaboración propia.

Nota: $\mathrm{EAG}=$ equivalente al ácido gálico; $\mathrm{EQ}=$ equivalente a quercetina; $\mathrm{T}-10=$ tortilla $+10 \%$ harina $B$. alicastrum; $\mathrm{T}-20=$ tortilla + $20 \%$ harina $B$. alicastrum; T-30 = tortilla $+30 \%$ harina $B$. alicastrum. Los resultados son promedios de tres repeticiones \pm desviación estándar; valores con diferentes letras dentro de cada columna son significativamente diferentes a $P \leq 0.05$.

\section{4. Propiedades físicas}

El color es el primer estímulo sensorial recibido por el consumidor, por lo que se considera una característica elemental para cualquier producto. En lo que respecta a este artículo, se reportan las coordenadas de color CIE obtenidas de la superficie de las tortillas, en donde, $L^{*}$ es referente a la luminosidad ( $0=$ negro y $100=$ blanco), $a^{*}$ va de verde a rojo y $b^{*}$ va de azul a amarillo (cuadro 5). Asimismo, se reporta que las tortillas adicionadas con harina de B. alicastrum resultaron con una disminución de los valores de $L^{*}$ y el ángulo de tono ( $\left.{ }^{\circ} \mathrm{Hue}\right)$, además del incremento de los valores de $a^{*}, b^{*}$ y cromaticidad. Conforme se incrementó la adición de harina de B. alicastrum, las tortillas de maíz presentaron una coloración marrón más obscura. Este cambio de coloración sería atribuible al fraccionamiento de tamaño de partícula de las harinas, que es un factor que debe tomarse en cuenta, ya que tiene influencia sobre el porcentaje de reflectancia de la harina, donde un menor tamaño de partícula origina mayor valor de reflectancia (Salinas-Moreno et al., 2010). Además, la cantidad de compuestos fenólicos y al contenido de minerales presentes en la harina de $B$. alicastrum explicarían el desarrollo de la coloración marrón (Pérez-Pacheco et al., 2014). El color marrón de las tortillas de maíz no representa una desventaja para su consumo, dado que en el mercado existen algunas marcas comerciales que tienen una coloración verde y obscura y son preferidas de forma considerable (Vázquez-Rodríguez et al., 2013).

En otro resultado, la incorporación de la harina de $B$. alicastrum afectó significativamente las propiedades físicas de las tortillas (cuadro 6). El valor del esfuerzo a la ruptura de la tortilla resultó mayor para T-10 y T-20 respecto al control y $\mathrm{T}-30$. 


\section{CUADRO 5}

Color de tortillas de maíz adicionado con harina de $B$. alicastrum en diferentes niveles

\begin{tabular}{|lccccc|}
\hline \multicolumn{1}{c}{ Muestra } & $L^{*}$ & $a^{*}$ & $b^{*}$ & ${ }^{\circ} H u e$ & Cromaticidad \\
\hline Control & $82.19 \pm 0.58 \mathrm{~d}$ & $0.97 \pm 0.10 \mathrm{a}$ & $20.10 \pm 0.85 \mathrm{a}$ & $87.22 \pm 0.19 \mathrm{c}$ & $20.12 \pm 0.86 \mathrm{a}$ \\
T-10 & $69.38 \pm 0.53 \mathrm{c}$ & $5.20 \pm 0.43 \mathrm{~b}$ & $23.67 \pm 0.76 \mathrm{~b}$ & $77.62 \pm 0.62 \mathrm{~b}$ & $24.23 \pm 0.84 \mathrm{~b}$ \\
T-20 & $64.98 \pm 0.60 \mathrm{~b}$ & $5.65 \pm 0.28 \mathrm{bc}$ & $23.63 \pm 0.51 \mathrm{~b}$ & $76.55 \pm 0.43 \mathrm{~b}$ & $24.29 \pm 0.55 \mathrm{~b}$ \\
T-30 & $58.21 \pm 0.90^{\mathrm{a}}$ & $6.22 \pm 0.42 \mathrm{c}$ & $22.73 \pm 1.06 \mathrm{~b}$ & $74.67 \pm 1.15 \mathrm{a}$ & $23.57 \pm 1.04 \mathrm{~b}$ \\
\hline
\end{tabular}

Fuente: elaboración propia.

Nota: $\mathrm{T}-10=$ tortilla $+10 \%$ harina $B$. alicastrum; $\mathrm{T}-20=$ tortilla $+20 \%$ harina $B$. alicastrum $; \mathrm{T}-30=$ tortilla $+30 \%$ harina $B$. alicastrum. Los resultados son promedios de tres repeticiones \pm desviación estándar; valores con diferentes letras dentro de cada columna son significativamente diferentes a $P \leq 0.05$.

La adición de harina de $B$. alicastrum produjo que disminuyera el porcentaje de elongación y un aumento del módulo de Young's de la tortilla de maíz. Este hecho explica que las tortillas de maíz adicionado con harina de B. alicastrum sean menos elásticas y más rígidas que la tortilla control. Este fenómeno puede atribuirse al uso de gomas en la harina comercial de maíz, lo cual es un factor importante al momento de elaborar tortillas, ya que influyen en su calidad como la elasticidad, humedad de las tortillas, además de prolongar su vida útil y ser más flexibles después de su cocción (Sánchez-Sandoval y Vázquez-Chávez, 2018). Estos resultados concuerdan con la rolabilidad, cuya medida subjetiva proporciona información sobre la calidad de las tortillas para formar un taco sin romperse. Cuando se incorporó 30\% de harina de B. alicastrum, se consiguió un valor más bajo de rolabilidad respecto al control, donde alrededor de $50 \%$ de las tortillas se rompen y con un grado de inflado medio. Las tortillas control T-10 y T-20 exhibieron características deseables respecto a rolabilidad (valores alrededor de 1) y completamente inflados. El grado de inflado de las tortillas fue el parámetro menos afectado, quizás se deba a la gelatinización del almidón de la harina de $B$. alicastrum durante la cocción, que contribuye que las tortillas no sean muy permeables, reteniendo al vapor de agua para la formación de la ampolla (Arámbula-Villa et al., 2001).

La pérdida de peso después de la cocción es un parámetro importante desde el punto de vista comercial en el rendimiento de las tortillas. El rango de la pérdida de peso de las tortillas que se acepta como adecuado debe oscilar entre 16 y 20\% (Arámbula-Villa et al., 2001). Con base en los resultados, la pérdida de peso por cocción no mostró diferencias significativas entre las tortillas.

Finalmente, la adición de harina de $B$. alicastrum no provocó cambios en los valores de espesor y diámetro de tortillas, lo que significa que el producto resultó uniforme.

\section{CUADRO 6}

Propiedades físicas de tortillas de maíz adicionado con harina de $B$. alicastrum en diferentes niveles

\begin{tabular}{lcccc} 
Muestra & Esfuerzo a la ruptura $(\mathrm{KPa})$ & Elongación $(\%)$ & Módulo Young's $(\mathrm{KPa})$ & Espesor $(\mathrm{mm})$ \\
\hline Control & $57.11 \pm 2.25 \mathrm{~b}$ & $9.26 \pm 0.54 \mathrm{~d}$ & $886.40 \pm 36.62 \mathrm{a}$ & $1.07 \pm 0.01 \mathrm{a}$ \\
T-10 & $133.68 \pm 2.38 \mathrm{~d}$ & $8.00 \pm 0.42 \mathrm{c}$ & $1945.40 \pm 126.63 \mathrm{c}$ & $1.11 \pm 0.04 \mathrm{a}$ \\
T-20 & $117.83 \pm 11.40 \mathrm{c}$ & $5.67 \pm 0.55 \mathrm{~b}$ & $2900.59 \pm 445.85 \mathrm{~d}$ & $1.11 \pm 0.02 \mathrm{a}$ \\
T-30 & $23.22 \pm 1.36 \mathrm{a}$ & $2.55 \pm 0.10 \mathrm{a}$ & $1409.00 \pm 117.78 \mathrm{~b}$ & $1.10 \pm 0.01 \mathrm{a}$ \\
\hline Muestra & Diámetro $(\mathrm{cm})$ & Pérdida por cocción $(\%)$ & Rolabilidad $^{\mathrm{A}}$ & Inflado $^{\mathrm{B}}$ \\
\hline Control & $12.37 \pm 0.12 \mathrm{a}$ & $30.41 \pm 0.61 \mathrm{a}$ & $1.13 \pm 0.06 \mathrm{a}$ & $1.53 \pm 0.06 \mathrm{a}$ \\
T-10 & $12.30 \pm 0.00 \mathrm{a}$ & $31.58 \pm 0.44 \mathrm{a}$ & $1.13 \pm 0.06 \mathrm{a}$ & $1.57 \pm 0.06 \mathrm{a}$ \\
T-20 & $12.40 \pm 0.10 \mathrm{a}$ & $32.26 \pm 1.09 \mathrm{a}$ & $1.33 \pm 0.06 \mathrm{~b}$ & $1.58 \pm 0.07 \mathrm{a}$ \\
T-30 & $12.47 \pm 0.06 \mathrm{a}$ & $31.33 \pm 0.58 \mathrm{a}$ & $2.97 \pm 0.06 \mathrm{c}$ & $1.93 \pm 0.12 \mathrm{~b}$ \\
\hline
\end{tabular}

Nota: $\mathrm{T}-10=$ tortilla $+10 \%$ harina $B$. alicastrum; $\mathrm{T}-20=$ tortilla $+20 \%$ harina $B$. alicastrum; $\mathrm{T}-30=$ tortilla $+30 \%$ harina $B$. alicastrum. Los resultados son promedios de tres repeticiones \pm desviación estándar; valores con diferentes letras dentro de cada columna son significativamente diferentes a $P \leq 0.05$. ARolabilidad: 1 a $5(1=0 \%$ rotura, $2=25 \%$ de rotura, $3=50 \%$ de rotura, $4=75 \%$ de rotura, 5 $=100 \%$ de rotura). ${ }^{\mathrm{B}}$ Inflado: 1 a $3[1=$ completamente inflado $(70-100 \%), 2=$ inflado medio $(30-70 \%), 3=$ no inflado $(0-30 \%)]$. 


\section{Conclusiones}

La incorporación de harina de $B$. alicastrum a la harina comercial de maíz afectó significativamente las propiedades tecnológicas funcionales de las mezclas de harina (IAA e ISA). En el aspecto nutricional, las tortillas adicionadas con harina de $B$. alicastrum obtuvieron mayor contenido de cenizas, fibra soluble y proteína total. Las tortillas de maíz enriquecidas con harina de B. alicastrum mostraron un aumento en el contenido de CFT, FT, actividad antioxidante, así como una coloración más marrón. Los valores del contenido de aminoácidos (triptófano, histidina, valina, isoleucina y leucina) de tortillas enriquecidas resultaron superiores a las tortillas control. La incorporación de 10 y $20 \%$ de harina de B. alicastrum produjo mayor resistencia a la ruptura y menos deformación de las tortillas; sin embargo, mantuvo sus características de rolabilidad e inflado respecto al control.

La harina de B. alicastrum es una alternativa para la fortificación de tortillas de maíz debido a su valor nutricional y funcional manteniendo la calidad del producto, opción que resultaría atractiva para la industria alimentaria.

\section{Prospectiva y sugerencias}

En México el maíz es considerado como el principal grano alimenticio que se importa para satisfacer los requerimientos la población; cualquier alteración de la oferta internacional significaría un riesgo de la seguridad alimentaria. Las tendencias actuales se han encaminado hacia el uso de materias primas no tradicionales de bajo costo que representen un valor agregado a sus fuentes originales y a los procesos de los cuales se elabora. El uso de ingredientes no tradicionales de la región es una opción de recurso disponible, económicamente viable y capaz de mejorar la seguridad alimentaria, como es el caso del fruto de B. alicastrum. No obstante, para su aprovechamiento se requiere el conocimiento de su cultivo, manejo, calidad y de la aplicación adecuada de la tecnología de alimentos. En la península de Yucatán, México, se han realizado esfuerzos por valorar las semillas de B. alicastrum como una alternativa de alimentación para combatir el hambre y se ha propuesto como una opción para sustituir a los granos importados por su alto valor nutricional y funcional, además de aprovecharse para el desarrollo de productos alimenticios como la tortilla. En el tema de prospectiva, las tortillas de maíz adicionadas con harina de $B$. alicastrum contribuyen en el interés por desarrollar nuevos productos alimenticios que ayuden a mejorar la salud de los consumidores.

La fortificación de las tortillas de maíz con harina de $B$. alicastrum en la formulación propuesta en este artículo representa una alternativa de mejora nutricional y antioxidante para este producto manteniendo su calidad. La información obtenida es fundamental para el manejo, procesamiento y fabricación de tortillas fortificadas en un nivel industrial sin causar un aumento en los costos de producción.

En estudios futuros se sugiere optimizar una mezcla de harinas (incluyendo B. alicastrum) para la elaboración de tortillas enriquecidas con mayor contenido de proteína y compuestos fenólicos utilizando un diseño simplex-centroide. Aunado a lo anterior, se sugiere un estudio sobre la evaluación del índice glucémico, digestibilidad de proteínas y almidón de las tortillas adicionadas con harina de $B$. alicastrum. Toda esta información contribuye a generar más evidencias científicas que promuevan el potencial de la harina de $B$. alicastrum, extender su cultivo y difundir sus propiedades.

\section{ReFerencias}

Anderson, R. A. (1982). Water absorption and solubility and amylograph characteristics of roll-cooked small grain products. Cereal Chemistry, 59(4), 265-269. 
Anton, A. A., Ross, K. A., Lukow, O. M., Fulcher, R. G., \& Arntfield, S. D. (2008). Influence of added bean flour (Phaseolus vulgaris L.) on some physical and nutritional properties of wheat flour tortillas. Food Chemistry, 109(1), 33-41.

AOAC. (1997). Official methods of analysis (15th ed.). USA: Association of Analytical Chemists.

Arámbula-Villa, G., Barrón-Ávila, L., González-Hernández, J., Moreno-Martínez, E., \& Luna-Bárcenas, G. (2001). Efecto del tiempo de cocimiento y reposo del grano de maíz (Zea mayz L.) nixtamalizado sobre las características fisicoquímicas, reológicas, estructurales y texturales del grano, masa y tortillas de maíz. Archivos Latinoamericanos de Nutrición, 51(2), 187-194.

Bello-Pérez, L. A., Osorio-Díaz, P., Agama-Acevedo, E., \& González-Soto, R. A. (2016). Functional and beneficial properties of corn tortilla. In Functional Properties of Traditional Foods (pp. 139-155). Boston: Springer.

Can-Cauich, C. A., Sauri-Duch, E., Betancur-Ancona, D., Chel-Guerrero, L., González-Aguilar, G. A., Cuevas-Glory, L. F., \& Moo-Huchin, V. M. (2017). Tropical fruit peel powders as functional ingredients: Evaluation of their bioactive compounds and antioxidant activity. Journal of Functional Foods, 37, 501-506.

Chuck-Hernández, C., Perez-Carrillo, E., Soria-Hernández, C., \& Serna-Saldívar, S. O. (2015). Functionality and organoleptic properties of maize tortillas enriched with five different soybean proteins. Cereal Chemistry, 92(4), 341-349.

Contreras-Jiménez, B., Morales-Sánchez, E., Reyes-Vega, M. L., \& Gaytán-Martínez, M. (2014). Propiedades funcionales de harinas de maíz nixtamalizado obtenidas por extrusión a baja temperatura. CyTA-Journal of Food, 12(3), 263-270.

Domínguez-Zarate, P. A., García-Martínez, I., Güemes-Vera, N., \& Totosaus, A. (2019). Textura, color y aceptación sensorial de tortillas y pan producidos con harina de ramón (Brosimum alicastrum) para incrementar la fibra dietética total. Ciencia \& Tecnología Agropecuaria, 20(3), 699-719.

FAO/WHO. (1991). Protein quality evaluations. Report of joint FAO/WHO expert consultation. Food and nutrition, 51. Rome: Food Agriculture Organization and the World Health Organization.

Flores-Farías, R., Martínez-Bustos, F., Salinas-Moreno y Ríos, E. (2002). Caracterización de harinas comerciales de maíz nixtamalizado. Agrociencia, 36, 557-567.

Gasca-Mancera, J. C. y Casas-Alencáster, N. B. (2007). Adición de harina de maíz nixtamalizado a masa fresca de maíz nixtamalizado. Efecto en las propiedades texturales de masa y tortilla. Revista Mexicana de Ingeniería Quimica, 6(3), 317-328.

GRAS report (2007). Maya nut (Brosimum alicastrum) and maya nut-derived ingredients for use in traditional foods (1-46 pp). Retrieved from https://www.doc-developpement-durable.org/file/Huiles-vegetales-noix/ Fiches_plantes/noyer\%20maya\%20-\%20Brosimum\%20alicastrum/MAYANUTGRASSTUDY.pdf

Liu, T., Hou, G. G., Book, S. L., \& Marquart, L. (2016). Effects of chemical leavening system and processing conditions on the opacity and other quality characteristics of whole-wheat flour tortillas. LWT-Food Science and Technology, 73, 123-130.

Moo-Huchin, V. M., Cabrera-Sierra, M. J., Estrada-León, R. J., Ríos-Soberanis, C. R., Betancur-Ancona, D., Chel-Guerrero, L., \& Pérez-Pacheco, E. (2015). Determination of some physicochemical and rheological characteristics of starch obtained from Brosimum alicastrum Swartz seeds. Food Hydrocolloids, 45, $48-54$.

Moo-Huchin, V. M., Canto-Pinto, J. C., Cuevas-Glory, L. F., Sauri-Duch, E., Pérez-Pacheco, E., \& Betancur-Ancona, D. (2019). Effect of extraction solvent on the phenolic compounds content and antioxidant activity of Ramon nut (Brosimum alicastrum). Chemical Papers, 73(7), 1647-1657. 
Moo-Huchin, V. M., Estrada-Mota, I., Estrada-León, R., Cuevas-Glory, L., Ortiz-Vázquez, E., y Vargas, M. D. L. V., \& Sauri-Duch, E. (2014). Determination of some physicochemical characteristics, bioactive compounds and antioxidant activity of tropical fruits from Yucatan, Mexico. Food Chemistry, 152, 508-515.

Ozer, H. K. (2017). Phenolic compositions and antioxidant activities of Maya nut (Brosimum alicastrum): Comparison with commercial nuts. International Journal of Food Properties, 20(11), 2772-2781.

Páramo-Calderón, D. E., Aparicio-Saguilán, A., Aguirre-Cruz, A., Carrillo-Ahumada, J., Hernández-Uribe, J. P., Acevedo-Tello, S., \& Torruco-Uco, J. G. (2019). Tortilla added with Moringa oleifera flour: Physicochemical, texture properties and antioxidant capacity. LWT-Food Science and Technology, 100, 409-415.

Pérez-Pacheco, E., Moo-Huchin, V. M., Estrada-León, R. J., Ortiz-Fernández, A., May-Hernández, L. H., Ríos-Soberanis, C. R., \& Betancur-Ancona, D. (2014). Isolation and characterization of starch obtained from Brosimum alicastrum Swarts Seeds. Carbohydrate Polymers, 101, 920-927.

Rendón-Villalobos, R., Ortíz-Sánchez, A., Solorza-Feria, J., \& Trujillo-Hernández, C. A. (2012). Formulation, physicochemical, nutritional and sensorial evaluation of corn tortillas supplemented with chia seed (Salvia hispanica L.). Czech Journal of Food Sciences, 30(2), 118-125.

Ríos-Soberanis, C. R., Moo-Huchin, V. M., Estrada-Leon, R. J., \& Perez-Pacheco, E. (2016). Comparative analysis between corn and ramon (Brosimum alicastrum) starches to be used as sustainable bio-based plastics. International Journal of Chemical and Molecular Engineering, 10(2), 211-214.

Ruíz-Ruíz, J., Martínez-Ayala, A., Drago, S., González, R., Betancur-Ancona, D., \& Chel-Guerrero, L. (2008). Extrusion of a hard-to-cook bean (Phaseolus vulgaris L.) and quality protein maize (Zea mays L.) flour blend. LWT-Food Science and Technology, 41(10), 1799-1807.

Saldívar, S. S., \& Chuck-Hernández, C. (2016). Tortillas. In Encyclopedia of Food and Health (pp. 319-325). United Kingdom: Elsevier.

Salinas-Moreno, Y., Gómez-Montiel, N. O., Cervantes-Martínez, J. E., Sierra-Macías, M., Palafox- Caballero, A., Betanzos-Mendoza, E., \& Coutiño-Estrada, B. (2010). Calidad nixtamalera y tortillera en maíces del trópico húmedo y sub-húmedo de México. Revista Mexicana de Ciencias Agrícolas, 1(4), 509-523.

Sánchez-Sandoval, D., \& Vázquez-Chávez, L. (2018). Vida de anaquel de tortillas de maíz nixtamalizado. Investigación y Desarrollo en Ciencia y Tecnología de alimentos, 3, 17-22.

Subiria-Cueto, R., Larqué-Saavedra, A., Reyes-Vega, M. L., De la Rosa, L. A., Santana-Contreras, L. E., Gaytán-Martínez, M., \& Martínez-Ruiz, N. R. (2019). Brosimum alicastrum Sw. (Ramón): An alternative to improve the nutritional properties and functional potential of the wheat flour tortilla. Foods, 8(12), 613.

Treviño-Mejía, D., Luna-Vital, D. A., Gaytán-Martínez, M., Mendoza, S., \& Loarca-Piña, G. (2016). Fortification of commercial nixtamalized maize (Zea mays L.) with common bean (Phaseolus vulgaris L.) increased the nutritional and nutraceutical content of tortillas without modifying sensory properties. Journal of Food Quality, 39(6), 569-579.

Vázquez-Rodríguez, J. A., Amaya-Guerra, C. A., Báez-González, J. G., Núñez-González, M. A., \& Figueroa-Cárdenas, J. D. (2013). Study of the fortification with bean and amaranth flours in nixtamalized maize torti1la. CyTA-Journal of Food, 11, 62-66.

Waliszewski, K. N., Pardio, V., \& Carreon, E. (2002). Physicochemical and sensory properties of corn tortillas made from nixtamalized corn flour fortified with spent soymilk residue (okara). Journal of Food Science, 67(8), 3194-3197. 